\title{
Relativistic Hartree-Fock and Dirac-Fock atomic structure and radiative parameter calculations in nine-times ionized xenon (Xe X)
}

\author{
E. Bokamba Motoumba a , S. Enzonga Yoca ${ }^{\mathrm{a}, \mathrm{b}}$, P. Palmeri ${ }^{\mathrm{c}}$, P. Quinet ${ }^{\mathrm{c}, \mathrm{d}, *}$ \\ a Faculté des Sciences et Techniques, Université Marien Ngouabi, BP 69 Brazzaville, Congo \\ ${ }^{\mathrm{b}}$ Conseil Africain et Malgache pour L'Enseignement Supérieur - CAMES, 01 BP 134 Ouagadougou 01, Burkina Faso \\ ' Physique Atomique et Astrophysique, Université de Mons, B-7000 Mons, Belgium \\ ${ }^{\mathrm{d}}$ IPNAS, Université de Liège, B-4000 Liège, Belgium
}

\section{A R T I C L E I N F O}

\section{Article history:}

Received 14 December 2018

Revised 17 January 2019

Accepted 17 January 2019

Available online 11 February 2019

\section{Keywords:}

Atomic structure

Transition probabilities

Oscillator strengths

Xe X spectrum

\begin{abstract}
A B S T R A C T
A new set of oscillator strengths and transition probabilities is reported for 92 radiative transitions of nine-times ionized xenon, Xe X, in the extreme ultraviolet region from 110 to $164 \AA$. They have been obtained by two different theoretical approaches, i.e. the pseudo-relativistic Hartree-Fock (HFR) and the fully relativistic multiconfiguration Dirac-Hartree-Fock (MCDHF) methods, which allowed us to estimate the precision of the final results. The eigenvector compositions are also given for the first time for all the experimentally known energy levels in Xe X.
\end{abstract}

(C) 2019 Published by Elsevier Ltd.

\section{Introduction}

In laboratory plasma physics, the radiative properties of noble gas ions present a particular interest since they can be injected into nuclear fusion installations, in the form of solidified pellets, for both fuel introduction and plasma diagnostics [1-3]. As an example, if xenon was inserted into ITER, it could be pumped out without leaving residuals on plasma-facing material, and thus would not be recycled in subsequent discharges. Moreover, the xenon atoms would strip to helium-like ions at the highest plasma temperatures. Consequently, the identification of emission lines and the knowledge of spectroscopic parameters from all ionization stages of xenon will greatly aid modelling of the plasma and facilitate the analysis of the spectra used for the estimation of physical conditions inside the fusion reactors such as densities and temperatures.

The spectroscopic properties of some xenon ions have already been the subject of different experimental and theoretical studies so far. In particular, as summarized in a recent review paper [4], pulsed high-current-high-voltage electrical discharges were widely used in La Plata, Argentina, to produce a low-pressure xenon plasma in order to identify many spectral lines of Xe III, Xe IV, Xe V, Xe VI, Xe VII, Xe VIII, and Xe IX in the UV, visible

\footnotetext{
* Corresponding author.

E-mail address: pascal.quinet@umons.ac.be (P. Quinet).
}

and infrared regions [5-11]. In these investigations, time-resolved experiments and relativistic Hartree-Fock calculations were also performed to obtain radiative lifetimes and transition probabilities. Moreover, the energy levels and spectral lines belonging to all xenon ionization stages for which experimental data were available before 2004, i.e. Xe I - Xe XI, Xe XIX, Xe XXV - Xe XXIX, Xe XLIII - Xe XLV, and Xe LI - Xe LIV, were compiled by Saloman [12].

As regards the radiative decay rates, we recently carried out large scale-calculations of lifetimes, oscillator strengths and transition probabilities in moderately charged xenon ions, from Xe V to Xe IX [13-16]. As a natural extension of these latter works, in the present paper, we report the theoretical calculations of electronic structure and decay rate parameters in nine-times ionized xenon, Xe X, for which no reliable data has been published so far. This ion is a bit more complicated to model than lower ionization stages due to the fact that, in addition to configurations of the type $4 \mathrm{~d}^{\mathrm{k}}$ and $4 \mathrm{~d}^{\mathrm{k}-1} \mathrm{nl}$, some low-lying energy levels have been identified as belonging to the core-excited $4 \mathrm{p}^{5} 4 \mathrm{~d}^{\mathrm{k}+1}$ configuration $(k=9)$. It is therefore obvious that a core-polarization model potential, like the one used in the HFR+CPOL approach $[17,18]$, is not sufficient to take such core-valence interactions into account which can only be considered by the explicit inclusion of core-excited configurations in the multiconfiguration expansions.

In view of the complete lack of experimental radiative parameters available for Xe X, the accuracy of the data obtained in our work has been assessed through the comparison between 
Table 1

Radial parameters adopted in the HFR calculations.

\begin{tabular}{|c|c|c|c|c|}
\hline Configuration & Parameter & HFR & Fitted & Ratio \\
\hline \multirow[t]{2}{*}{$4 d^{9}$} & $\mathrm{E}_{\mathrm{av}}$ & 9465 & 9684 & \\
\hline & $\zeta_{4 \mathrm{~d}}$ & 6473 & 6692 & 1.034 \\
\hline \multirow[t]{9}{*}{$4 d^{8} 5 p$} & $\mathrm{E}_{\mathrm{av}}$ & 680,178 & 675,157 & \\
\hline & $\mathrm{F}^{2}(4 \mathrm{~d}, 4 \mathrm{~d})$ & 123,794 & 105,003 & 0.848 \\
\hline & $\mathrm{F}^{4}(4 \mathrm{~d}, 4 \mathrm{~d})$ & 83,122 & 74,435 & 0.895 \\
\hline & $\alpha$ & 0 & 13 & \\
\hline & $\zeta_{4 \mathrm{~d}}$ & 6691 & 6869 & 1.027 \\
\hline & $\zeta_{5 p}$ & 14,645 & 15,867 & 1.083 \\
\hline & $\mathrm{F}^{2}(4 \mathrm{~d}, 5 \mathrm{p})$ & 47,918 & 41,940 & 0.875 \\
\hline & $G^{1}(4 d, 5 p)$ & 14,216 & 12,845 & 0.904 \\
\hline & $G^{3}(4 d, 5 p)$ & 14,157 & 14,359 & 1.014 \\
\hline \multirow[t]{11}{*}{$4 d^{8} 4 f$} & $\mathrm{E}_{\mathrm{av}}$ & 734,757 & 730,029 & \\
\hline & $\mathrm{F}^{2}(4 \mathrm{~d}, 4 \mathrm{~d})$ & 122,608 & 110,426 & 0.901 \\
\hline & $\mathrm{F}^{4}(4 \mathrm{~d}, 4 \mathrm{~d})$ & 82,235 & 85,930 & 1.045 \\
\hline & $\alpha$ & 0 & 22 & \\
\hline & $\zeta_{4 \mathrm{~d}}$ & 6559 & 6619 & 1.009 \\
\hline & $\zeta_{4 \mathrm{f}}$ & 439 & 439 & 1.000 \\
\hline & $F^{2}(4 d, 4 f)$ & 102,751 & 93,386 & 0.909 \\
\hline & $\mathrm{F}^{4}(4 \mathrm{~d}, 4 \mathrm{f})$ & 66,053 & 64,240 & 0.973 \\
\hline & $G^{1}(4 d, 4 f)$ & 122,956 & 102,588 & 0.834 \\
\hline & $G^{3}(4 d, 4 f)$ & 76,946 & 56,114 & 0.729 \\
\hline & $G^{5}(4 d, 4 f)$ & 54,360 & 38,360 & 0.706 \\
\hline \multirow[t]{2}{*}{$4 p^{5} 4 d^{10}$} & Eav & 769,881 & 737,711 & \\
\hline & $\zeta_{4 \mathrm{p}}$ & 66,597 & 87,061 & 1.307 \\
\hline \multirow[t]{4}{*}{$4 d^{8} 5 p-4 d^{8} 4 f$} & $R^{2}(4 d, 5 p ; 4 d, 4 f)$ & $-11,675$ & -8918 & 0.764 \\
\hline & $R^{4}(4 d, 5 p ; 4 d, 4 f)$ & -3557 & -2718 & 0.764 \\
\hline & $\mathrm{R}^{1}(4 \mathrm{~d}, 5 \mathrm{p} ; 4 \mathrm{~d}, 4 \mathrm{f})$ & -3726 & -2845 & 0.764 \\
\hline & $R^{3}(4 d, 5 p ; 4 d, 4 f)$ & -714 & -545 & 0.764 \\
\hline \multirow[t]{2}{*}{$4 d^{8} 5 p-4 p^{5} 4 d^{10}$} & $\mathrm{R}^{1}(4 \mathrm{p}, 5 \mathrm{p} ; 4 \mathrm{~d}, 4 \mathrm{~d})$ & 4136 & 3516 & 0.850 \\
\hline & $\mathrm{R}^{3}(4 \mathrm{p}, 5 \mathrm{p} ; 4 \mathrm{~d}, 4 \mathrm{~d})$ & 7029 & 5975 & 0.850 \\
\hline \multirow[t]{2}{*}{$4 d^{8} 4 f-4 p^{5} 4 d^{10}$} & $\mathrm{R}^{1}(4 \mathrm{p}, 4 \mathrm{f} ; 4 \mathrm{~d}, 4 \mathrm{~d})$ & 138,421 & 118,048 & 0.853 \\
\hline & $\mathrm{R}^{3}(4 \mathrm{p}, 4 \mathrm{f} ; 4 \mathrm{~d}, 4 \mathrm{~d})$ & 85,758 & 73,137 & 0.853 \\
\hline
\end{tabular}

two independent theoretical approaches, i.e. the pseudo-relativistic Hartree-Fock (HFR) [19] and the fully relativistic multiconfiguration Dirac-Hartree-Fock (MCDHF) [20] methods, both of them including explicitly the most important intravalence and core-valence electron correlations. This allowed us to compute, for the first time, the oscillator strengths and transition probabilities for a set of 92 spectral lines involving experimentally known energy levels in ninetimes ionized xenon, in the EUV wavelength range from 110 to $164 \AA$.

\section{Theoretical methods}

\subsection{Pseudo-relativistic Hartree-Fock calculations}

In a first step, the pseudo-relativistic Hartree-Fock (HFR) method, as described by Cowan [19], was applied for modelling the atomic structure and computing the radiative transition rates in $\mathrm{Xe} \mathrm{X}$. The electron correlation was considered by retaining explicitly in the physical model the following 13 even-parity and 11 odd-parity configurations, respectively: $4 d^{9}+4 d^{8} 5 s+4 d^{8} 6 s+4 d^{8} 7 s+4 d^{8} 5 d+4 d^{8} 6 d+4 d^{7} 5 s^{2}+4 d^{7} 5 p^{2}+4$ $d^{7} 5 d^{2}+4 d^{7} 5 s 6 s+4 d^{7} 5 s 5 d+4 d^{7} 5 s 6 d+4 p^{5} 4 d^{9} 5 p$, and $4 d^{8} 5 p+4 d^{8}$ $6 p+4 d^{8} 7 p+4 d^{8} 4 f+4 d^{8} 5 f+4 d^{8} 6 f+4 d^{7} 5 s 5 p+4 d^{7} 5 s 6 p+4 d^{7} 5 p 5 d$ $+4 d^{7} 5 p 6 s+4 p^{5} 4 d^{10}$. These sets of configurations are expected to take the most important intravalence and core-valence interactions into account for the lowest configurations in both parities and thus for the transitions considered in the present work, i.e. $4 d^{9}-4 d^{8} 5 p, 4 d^{9}-4 d^{8} 4 f$ and $4 d^{9}-4 p^{5} 4 d^{10}$. In particular, in view of their low average energies, it was necessary to explicitly include the $4 p^{5} 4 d^{10}$ and $4 p^{5} 4 d^{9} 5 p$ core-excited configurations in the physical model, preventing to use a core-polarization pseudopotential, as developed in the HFR+CPOL method depending on two parameters, i.e. the dipole polarizability and the cut-off radius of the ionic core $[17,18]$. However, it was recently shown that core-polarization effects were not very large in the case of Xe IX, the HFR+CPOL transition rates being found to be only a few percent different from the HFR values [16]. Therefore, instead of using the HFR+CPOL approach, we used the original HFR method of Cowan [19] in which the most important core-valence correlation effects were taken into account by explicitly including some configurations with one hole in the $4 p$ subshell.

In order to simulate the interactions with more distant configurations, the HFR method was then combined with a well-known semi-empirical fitting procedure of the calculated eigenvalues to all the available experimental energy levels taken from [12]. Thus, the average energies $\left(E_{a v}\right)$, Slater integrals $\left(F^{k}, G^{k}, R^{k}\right)$, spin-orbit parameters $\left(\zeta_{n l}\right)$ and effective interaction operators $(\alpha)$ belonging to the $4 \mathrm{~d}^{9}, 4 \mathrm{~d}^{8} 5 \mathrm{p}, 4 \mathrm{~d}^{8} 4 \mathrm{f}$, and $4 \mathrm{p}^{5} 4 \mathrm{~d}^{10}$ configurations were adjusted to minimize the differences between computed and experimental energy levels, leading to mean deviations of $0 \mathrm{~cm}^{-1}$ for the even parity (2 levels) and $162 \mathrm{~cm}^{-1}$ for the odd parity (60 levels). The parameters adopted in the HFR calculations are given in Table 1 . For all the other configurations than those listed in this table, the ab initio HFR values of the Slater integrals were reduced by $10 \%$, as recommended by Cowan [19], while the spin-orbit parameters, computed by the Blume-Watson method, were used unscaled. This scaling of the Slater electrostatic interaction integrals is a well-established practice allowing to consider the effect of farinteracting configurations non-explicitly included in the physical model. The detailed comparison between HFR and experimental energy levels is given in Table 2, in which the main LS eigenvector components are also reported. We note that a large majority of the levels considered in our work appear to be strongly mixed, the average LS purity being found equal to $40 \%$ for the 60 odd-parity levels, with values as low as $20-30 \%$ for about fifteen levels.

\subsection{Fully relativistic Dirac-Hartree-Fock calculations}

In order to calculate the oscillator strengths of the $\mathrm{E} 1$ transition arrays $4 \mathrm{~d}^{9} \mathrm{~J}^{\Pi}=3 / 2,5 / 2-\left(4 \mathrm{p}^{5} 4 \mathrm{~d}^{10}+4 \mathrm{~d}^{8} 5 \mathrm{p}+4 \mathrm{~d}^{8} 4 \mathrm{f}\right) \mathrm{J}^{\Pi}=1 / 2^{\circ}$ - $7 / 2^{\circ}$, the fully relativistic multiconfiguration Dirac-Hartree-Fock (MCDHF) method [20] was used as implemented in the GRASP2K atomic structure computer package [21]. In this method, the atomic state function (ASF), $\Psi$, is represented by a superposition of configuration state functions (CSF), $\Phi$, with the same parity, $\Pi$, total angular momentum, $\mathrm{J}$, and total magnetic quantum numbers, $\mathrm{M}_{\mathrm{J}}$, forming a basis set of the representation, $\left\{\Phi_{\mathrm{k}}\right\}$, as

$\Psi\left(\Pi J M_{J}\right)=\sum_{k} c_{k} \Phi_{k}\left(\gamma_{k} \Pi J M_{J}\right)$

where $\left\{c_{k}\right\}$ are the mixing coefficients, $\gamma_{k}$ represent all the other quantum numbers needed to uniquely specify the CSF built from one-electron spin-orbitals $\phi_{n \kappa m}(r, \theta, \varphi)$, of the form:

$\varphi_{n \kappa m}(r, \theta, \phi)=\frac{1}{r}\left(\begin{array}{c}P_{n \kappa}(r) \chi_{\kappa m}(\theta, \phi) \\ i Q_{n \kappa}(r) \chi_{-\kappa m}(\theta, \phi)\end{array}\right)$.

$P_{n \kappa}(r)$ and $Q_{n \kappa}(r)$ are, respectively, the large and the small radial components of the wave functions, and the angular functions $\chi_{\kappa m}(\theta, \varphi)$ are the spinor spherical harmonics [20]. The quantum number $\kappa$ is given by:

$\kappa= \pm\left(j+\frac{1}{2}\right)$

where $\kappa=-(j+1 / 2) a$, with a defined so that

$l=j-\frac{1}{2} a \quad ; \quad a= \pm 1$

The radial wavefunctions $P_{n \kappa}(r)$ and $Q_{n \kappa}(r)$ are numerically represented on a logarithmic grid and are required to be orthonormal within each $\kappa$ symmetry. In the MCDHF variational procedure, the 
Table 2

Comparison between the calculated and available experimental energy levels in Xe X, together with the corresponding LS-coupling components.

\begin{tabular}{|c|c|c|c|c|}
\hline $\begin{array}{l}\mathrm{E}_{\mathrm{EXP}^{\mathrm{a}}} \\
\left(\mathrm{cm}^{-1}\right)\end{array}$ & $\begin{array}{l}\mathrm{E}_{\mathrm{HFR}} \\
\left(\mathrm{cm}^{-1}\right)\end{array}$ & $\begin{array}{l}\mathrm{E}_{\mathrm{MCDHF}} \\
\left(\mathrm{cm}^{-1}\right)\end{array}$ & $\mathrm{J}$ & $\begin{array}{l}\text { Composition }{ }^{\mathrm{b}} \\
(\%)\end{array}$ \\
\hline \multicolumn{5}{|c|}{ Even parity } \\
\hline 0 & 0 & 0 & $5 / 2$ & $1004 d^{9}{ }^{2} \mathrm{D}$ \\
\hline 16,725 & 16,725 & 16,522 & $3 / 2$ & $1004 d^{9}{ }^{2} \mathrm{D}$ \\
\hline \multicolumn{5}{|c|}{ Odd parity } \\
\hline 629,040 & 629,139 & 621,451 & $7 / 2$ & $674 d^{8} 5 p\left({ }^{3} F\right)^{4} D+164 d^{8} 5 p\left({ }^{3} F\right)^{4} F+84 d^{8} 5 p\left({ }^{3} F\right)^{2} F$ \\
\hline 629,234 & 629,036 & 637,202 & $3 / 2$ & $724 \mathrm{p}^{5} 4 \mathrm{~d}^{10}{ }^{2} \mathrm{P}+214 \mathrm{~d}^{8} 4 \mathrm{f}(1 \mathrm{G})^{2} \mathrm{P}+54 \mathrm{~d}^{8} 4 \mathrm{f}\left({ }^{1} \mathrm{D}\right)^{2} \mathrm{P}$ \\
\hline 644,130 & 643,967 & 646,576 & $3 / 2$ & $274 d^{8} 5 p\left({ }^{1} D\right)^{2} D+194 d^{8} 5 p\left({ }^{3} P\right)^{4} P+184 d^{8} 5 p\left({ }^{1} D\right)^{2} P$ \\
\hline 646,494 & 646,486 & 639,516 & $5 / 2$ & $284 d^{8} 5 p\left({ }^{3} F\right)^{4} G+274 d^{8} 5 p\left({ }^{3} F\right)^{4} F+244 d^{8} 5 p\left({ }^{3} F\right)^{4} D$ \\
\hline 646,880 & 646,813 & 639,819 & $7 / 2$ & $644 d^{8} 5 p\left({ }^{3} F\right)^{4} G+154 d^{8} 5 p\left({ }^{3} F\right)^{2} G+114 d^{8} 5 p\left({ }^{3} F\right)^{4} F$ \\
\hline 654,245 & 654,344 & 647,294 & $3 / 2$ & $364 d^{8} 5 p\left({ }^{3} F\right)^{4} D+294 d^{8} 5 p\left({ }^{3} P\right)^{4} D+174 d^{8} 5 p\left({ }^{3} P\right)^{4} P$ \\
\hline 656,520 & 656,408 & 649,461 & $1 / 2$ & $374 d^{8} 5 p\left({ }^{3} P\right)^{4} D+244 d^{8} 5 p\left({ }^{3} F\right)^{4} D+134 d^{8} 5 p\left({ }^{3} P\right)^{2} P$ \\
\hline 657,645 & 657,655 & 651,295 & $5 / 2$ & $344 d^{8} 5 p\left({ }^{3} F\right)^{4} G+174 d^{8} 5 p\left({ }^{3} F\right)^{2} D+174 d^{8} 5 p\left({ }^{3} P\right)^{4} P$ \\
\hline 658,993 & 659,185 & 652,411 & $7 / 2$ & $554 d^{8} 5 p\left({ }^{3} F\right)^{2} F+194 d^{8} 5 p\left({ }^{3} F\right)^{4} D+134 d^{8} 5 p\left({ }^{3} F\right)^{4} F$ \\
\hline 662,160 & 662,203 & 655,776 & $3 / 2$ & $264 d^{8} 5 p\left({ }^{3} P\right)^{4} P+134 d^{8} 5 p\left({ }^{3} F\right)^{4} D+124 d^{8} 5 p\left({ }^{3} F\right)^{4} F$ \\
\hline 664,256 & 664,472 & 658,965 & $5 / 2$ & $564 d^{8} 5 p\left({ }^{3} F\right)^{2} D+134 d^{8} 5 p\left({ }^{3} P\right)^{4} P+124 d^{8} 5 p\left({ }^{3} F\right)^{4} D$ \\
\hline 668,525 & 668,586 & 661,721 & $5 / 2$ & $264 d^{8} 5 p\left({ }^{1} D\right)^{2} F+234 d^{8} 5 p\left({ }^{3} F\right)^{4} F+164 d^{8} 5 p\left({ }^{3} F\right)^{4} D$ \\
\hline 669,531 & 669,657 & 663,688 & $7 / 2$ & $334 d^{8} 5 p\left({ }^{1} D\right)^{2} F+294 d^{8} 5 p(1 G)^{2} F+194 d^{8} 5 p\left({ }^{3} F\right)^{2} G$ \\
\hline 671,045 & 671,335 & 665,459 & $5 / 2$ & $284 d^{8} 5 p\left({ }^{3} F\right)^{2} F+234 d^{8} 5 p\left({ }^{1} D\right)^{2} D+174 d^{8} 5 p\left({ }^{3} P\right)^{2} D$ \\
\hline 672,762 & 672,810 & 666,208 & $7 / 2$ & $494 d^{8} 5 p\left({ }^{3} F\right)^{4} F+144 d^{8} 5 p\left({ }^{3} F\right)^{4} G+104 d^{8} 5 p(1 G)^{2} F$ \\
\hline 674,159 & 673,963 & 668,501 & $3 / 2$ & $184 d^{8} 5 p\left({ }^{3} P\right)^{2} P+174 d^{8} 5 p\left({ }^{3} F\right)^{4} F+174 d^{8} 5 p\left({ }^{3} P\right)^{4} D$ \\
\hline 675,652 & 675,649 & 669,892 & $5 / 2$ & $204 d^{8} 5 p\left({ }^{1} D\right)^{2} D+164 d^{8} 5 p\left({ }^{1} D\right)^{2} F+134 d^{8} 5 p\left({ }^{3} F\right)^{4} F$ \\
\hline 675,878 & 675,727 & 671,198 & $7 / 2$ & $344 d^{8} 5 p(1 G)^{2} F+254 d^{8} 5 p\left({ }^{3} F\right)^{2} G+194 d^{8} 5 p\left({ }^{3} F\right)^{2} F$ \\
\hline 676,893 & 676,999 & 678,491 & $7 / 2$ & $394 d^{8} 4 f\left({ }^{3} F\right)^{4} F+304 d^{8} 4 f\left({ }^{3} P\right)^{4} F+174 d^{8} 4 f\left({ }^{3} F\right)^{4} D$ \\
\hline 677,421 & 677,240 & 672,775 & $3 / 2$ & $314 d^{8} 5 p\left({ }^{1} D\right)^{2} P+294 d^{8} 5 p\left({ }^{3} F\right)^{4} F+164 d^{8} 5 p\left({ }^{3} P\right)^{2} P$ \\
\hline 677,704 & 677,508 & 673,473 & $1 / 2$ & $334 d^{8} 5 p\left({ }^{3} P\right)^{4} D+314 d^{8} 5 p\left({ }^{1} D\right)^{2} P+214 d^{8} 5 p\left({ }^{3} F\right)^{4} D$ \\
\hline 678,351 & 678,016 & 679,628 & $5 / 2$ & $304 d^{8} 4 f\left({ }^{3} F\right)^{4} F+264 d^{8} 4 f\left({ }^{3} P\right)^{4} F+214 d^{8} 4 f\left({ }^{3} F\right)^{4} D$ \\
\hline 681,425 & 681,432 & 675,499 & $1 / 2$ & $364 d^{8} 5 p\left({ }^{3} F\right)^{4} D+344 d^{8} 5 p\left({ }^{3} P\right)^{2} P+74 d^{8} 5 p\left({ }^{1} D\right)^{2} P$ \\
\hline 682,437 & 682,479 & 684,251 & $3 / 2$ & $334 d^{8} 4 f\left({ }^{3} F\right)^{4} D+224 d^{8} 4 f\left({ }^{3} F\right)^{4} F+224 d^{8} 4 f\left({ }^{3} P\right){ }^{4} F$ \\
\hline 682,838 & 683,059 & 677,482 & $5 / 2$ & $364 d^{8} 5 p\left({ }^{3} F\right)^{2} F+154 d^{8} 5 p\left({ }^{3} P\right)^{4} P+124 d^{8} 5 p\left({ }^{3} P\right)^{2} D$ \\
\hline 682,998 & 682,960 & 678,481 & $3 / 2$ & $544 d^{8} 5 p\left({ }^{3} F\right)^{2} D+164 d^{8} 5 p\left({ }^{1} D\right)^{2} D+104 d^{8} 5 p\left({ }^{3} P\right)^{4} D$ \\
\hline 684,240 & 684,212 & 679,236 & $7 / 2$ & $504 d^{8} 5 p\left({ }^{3} P\right)^{4} D+4 d^{8} 5 p\left({ }^{3} F\right)^{2} G+4 d^{8} 5 p\left({ }^{3} F\right)^{4} G$ \\
\hline 684,807 & 685,212 & 685,328 & $1 / 2$ & $364 d^{8} 4 f(1 G)^{2} P+164 d^{8} 4 f\left({ }^{3} F\right)^{2} P+124 d^{8} 4 f\left({ }^{3} F\right)^{4} D$ \\
\hline 686,273 & 686,020 & 687,536 & $5 / 2$ & $444 d^{8} 4 f\left({ }^{3} F\right)^{4} D+234 d^{8} 4 f\left({ }^{3} F\right)^{4} P+134 d^{8} 4 f\left({ }^{3} P\right)^{4} F$ \\
\hline 687,770 & 687,707 & 688,696 & $7 / 2$ & $724 d^{8} 4 f\left({ }^{3} F\right)^{4} D+104 d^{8} 4 f\left({ }^{3} P\right)^{4} F+64 d^{8} 4 f\left({ }^{1} D\right)^{2} F$ \\
\hline 688,121 & 688,119 & 683,566 & $3 / 2$ & $224 d^{8} 5 p\left({ }^{3} P\right)^{2} D+214 d^{8} 5 p\left({ }^{3} P\right)^{2} P+144 d^{8} 5 p\left({ }^{1} D\right)^{2} P$ \\
\hline 689,190 & 688,913 & 690,908 & $3 / 2$ & $264 d^{8} 4 f\left({ }^{3} P\right){ }^{4} F+164 d^{8} 4 f\left({ }^{3} F\right)^{4} F+164 d^{8} 4 f\left({ }^{3} F\right)^{4} P$ \\
\hline 690,757 & 690,553 & 684,912 & $5 / 2$ & $524 d^{8} 5 p(1 G)^{2} F+164 d^{8} 5 p\left({ }^{1} D\right)^{2} D+104 d^{8} 5 p\left({ }^{3} F\right)^{4} F$ \\
\hline 691,306 & 691,128 & 686,637 & $3 / 2$ & $194 d^{8} 5 p\left({ }^{3} P\right)^{4} S+154 d^{8} 5 p\left({ }^{3} P\right)^{4} D+154 d^{8} 5 p\left({ }^{1} D\right)^{2} D$ \\
\hline 694,056 & 693,940 & 689,938 & $5 / 2$ & $444 d^{8} 5 p\left({ }^{3} P\right)^{4} D+344 d^{8} 5 p\left({ }^{3} P\right)^{2} D+104 d^{8} 5 p(1 G)^{2} F$ \\
\hline 695,239 & 695,273 & 692,079 & $1 / 2$ & $574 d^{8} 5 p\left({ }^{3} P\right)^{2} S+114 d^{8} 5 p\left({ }^{3} P\right)^{2} P+94 d^{8} 5 p\left({ }^{3} P\right)^{4} P$ \\
\hline 696,975 & 697,042 & 692,202 & $3 / 2$ & $474 d^{8} 5 p\left({ }^{3} P\right)^{2} D+94 d^{8} 5 p\left({ }^{1} D\right)^{2} P+84 d^{8} 5 p\left({ }^{1} D\right)^{2} D$ \\
\hline 697,440 & 697,688 & 699,982 & $3 / 2$ & $304 d^{8} 4 f\left({ }^{3} F\right)^{4} P+174 d^{8} 4 f\left({ }^{3} F\right)^{4} S+104 d^{8} 4 f\left({ }^{3} F\right)^{4} D$ \\
\hline 698,751 & 699,131 & 701,179 & $5 / 2$ & $294 d^{8} 4 f\left({ }^{1} D\right)^{2} F+284 d^{8} 4 f\left({ }^{3} F\right)^{4} \mathrm{P}+84 d^{8} 4 f\left({ }^{3} P\right)^{2} F$ \\
\hline 701,155 & 701,098 & 703,270 & $5 / 2$ & $314 d^{8} 4 f\left({ }^{3} F\right)^{4} P+144 d^{8} 4 f\left({ }^{3} F\right)^{4} D+64 d^{8} 4 f\left({ }^{1} D\right)^{2} F$ \\
\hline 701,735 & 702,031 & 696,646 & $5 / 2$ & $214 d^{8} 5 p(1 G)^{2} F+204 d^{8} 5 p\left({ }^{1} D\right)^{2} D+124 d^{8} 5 p\left({ }^{3} F\right)^{2} F$ \\
\hline 702,652 & 702,575 & 704,098 & $1 / 2$ & $764 d^{8} 4 f\left({ }^{3} F\right)^{4} P+94 d^{8} 4 f\left({ }^{3} F\right)^{4} D+54 d^{8} 4 f(1 G)^{2} P$ \\
\hline 703,997 & 703,772 & 700,712 & $7 / 2$ & $524 d^{8} 5 p(1 G)^{2} G+174 d^{8} 5 p(1 G)^{2} F+164 d^{8} 5 p\left({ }^{1} D\right)^{2} F$ \\
\hline 705,669 & 705,660 & 701,739 & $1 / 2$ & $364 d^{8} 5 p\left({ }^{1} D\right)^{2} P+364 d^{8} 5 p\left({ }^{3} P\right)^{2} P+104 d^{8} 5 p\left({ }^{3} P\right)^{2} S$ \\
\hline 708,748 & 708,404 & 711,811 & $7 / 2$ & $194 d^{8} 4 f\left({ }^{3} F\right)^{2} G+184 d^{8} 4 f\left({ }^{1} D\right)^{2} F+144 d^{8} 4 f\left({ }^{1} D\right)^{2} G$ \\
\hline 711,392 & 711,380 & 714,253 & $5 / 2$ & $394 d^{8} 4 f(1 G)^{2} D+204 d^{8} 4 f\left({ }^{3} F\right)^{2} D+104 d^{8} 4 f\left({ }^{3} F\right)^{4} D$ \\
\hline 713,643 & 713,471 & 717,071 & $5 / 2$ & $424 d^{8} 4 f\left({ }^{3} F\right)^{4} G+284 d^{8} 4 f\left({ }^{3} P\right)^{4} G+104 d^{8} 4 f\left({ }^{3} P\right)^{4} F$ \\
\hline 721,870 & 721,894 & 725,433 & $5 / 2$ & $404 d^{8} 4 f\left({ }^{3} F\right)^{4} F+284 d^{8} 4 f\left({ }^{3} P\right)^{4} F+74 d^{8} 4 f\left({ }^{3} P\right)^{4} G$ \\
\hline 723,216 & 723,557 & 721,967 & $1 / 2$ & $624 d^{8} 5 p\left({ }^{1} S\right)^{2} P+84 d^{8} 5 p\left({ }^{1} D\right)^{2} P+74 d^{8} 4 f\left({ }^{1} D\right)^{2} P$ \\
\hline 725,785 & 726,204 & 729,059 & $1 / 2$ & $364 d^{8} 4 f\left({ }^{3} P\right)^{4} D+174 d^{8} 4 f\left({ }^{1} D\right)^{2} P+154 d^{8} 4 f\left({ }^{3} F\right)^{2} S$ \\
\hline 737,104 & 737,429 & 741,773 & $1 / 2$ & $464 d^{8} 4 f\left({ }^{3} P\right)^{4} D+244 d^{8} 4 f\left({ }^{3} F\right)^{2} P+184 p^{5} 4 d^{10}{ }^{2} P$ \\
\hline 745,212 & 745,018 & 742,492 & $3 / 2$ & $554 d^{8} 5 p\left({ }^{1} S\right)^{2} P+174 d^{8} 4 f\left({ }^{1} D\right)^{2} D+94 d^{8} 4 f(1 G)^{2} D$ \\
\hline 749,681 & 748,896 & 756,115 & $3 / 2$ & $204 d^{8} 4 f\left({ }^{1} D\right)^{2} D+164 d^{8} 4 f\left({ }^{1} D\right)^{2} P+154 d^{8} 5 p\left({ }^{1} S\right)^{2} P$ \\
\hline 753,489 & 753,740 & 762,165 & $1 / 2$ & $444 d^{8} 4 f\left({ }^{1} D\right)^{2} P+214 d^{8} 4 f\left({ }^{3} F\right)^{2} P+154 p^{5} 4 d^{10}{ }^{2} P$ \\
\hline 864,592 & 864,495 & 885,636 & $5 / 2$ & $524 d^{8} 4 f\left({ }^{3} F\right)^{2} D+234 d^{8} 4 f(1 G)^{2} D+94 d^{8} 4 f\left({ }^{1} D\right)^{2} D$ \\
\hline 870,470 & 871,402 & 889,355 & $7 / 2$ & $354 d^{8} 4 f\left({ }^{3} F\right)^{2} F+324 d^{8} 4 f\left({ }^{3} P\right)^{2} F+134 d^{8} 4 f\left({ }^{1} D\right)^{2} F$ \\
\hline 874,794 & 874,699 & 894,194 & $3 / 2$ & $334 d^{8} 4 f\left({ }^{3} F\right)^{2} D+174 d^{8} 4 f(1 G)^{2} P+154 d^{8} 4 f(1 G)^{2} D$ \\
\hline 881,539 & 881,780 & 901,530 & $3 / 2$ & $264 d^{8} 4 f(1 G)^{2} P+224 d^{8} 4 f\left({ }^{3} F\right)^{2} D+204 d^{8} 4 f\left({ }^{3} F\right)^{2} P$ \\
\hline 887,203 & 887,474 & 905,905 & $5 / 2$ & $344 d^{8} 4 f\left({ }^{3} F\right)^{2} F+304 d^{8} 4 f\left({ }^{3} P\right)^{2} F+144 d^{8} 4 f\left({ }^{1} D\right)^{2} F$ \\
\hline 924,721 & 924,709 & 934,573 & $1 / 2$ & $424 \mathrm{p}^{5} 4 \mathrm{~d}^{10}{ }^{2} \mathrm{P}+294 \mathrm{~d}^{8} 4 \mathrm{f}(1 \mathrm{G})^{2} \mathrm{P}+194 \mathrm{~d}^{8} 4 \mathrm{f}\left({ }^{3} \mathrm{~F}\right)^{2} \mathrm{P}$ \\
\hline
\end{tabular}

a Experimental energy level values taken from [12].

b HFR composition in LS-coupling. Only the first three components greater than $5 \%$ are given. 
Table 3

HFR and MCDHF oscillator strengths (log gf) and transition probabilities (gA) in Xe X.

\begin{tabular}{|c|c|c|c|c|c|c|c|c|c|c|}
\hline \multirow[t]{2}{*}{$\lambda(\AA)^{\mathrm{a}}$} & \multirow{2}{*}{$\begin{array}{l}\text { Lower level }^{\text {b }} \\
\text { E }\left(\mathrm{cm}^{-1}\right)\end{array}$} & \multicolumn{3}{|c|}{ Upper level $^{\mathrm{b}}$} & \multicolumn{3}{|l|}{ HFR } & \multicolumn{3}{|c|}{ MCDHF } \\
\hline & & $\mathrm{J}$ & $\mathrm{E}\left(\mathrm{cm}^{-1}\right)$ & $\mathrm{J}$ & $\log g f$ & $\mathrm{gA}\left(\mathrm{s}^{-1}\right)$ & $\mathrm{CF}^{\mathrm{c}}$ & $\log g f$ & $\mathrm{gA}\left(\mathrm{s}^{-1}\right)$ & $\mathrm{B} / \mathrm{C}^{\mathrm{d}}$ \\
\hline 110.133 & 16,725 & $3 / 2$ & 924,721 & $1 / 2$ & 0.84 & $3.84 \mathrm{E}+12$ & 0.92 & 0.74 & $3.10 \mathrm{E}+12$ & 1.05 \\
\hline 112.714 & 0 & $5 / 2$ & 887,203 & $5 / 2$ & -0.71 & $1.03 \mathrm{E}+11$ & 0.05 & -1.17 & $3.68 \mathrm{E}+10$ & 0.94 \\
\hline 113.438 & 0 & $5 / 2$ & 881,539 & $3 / 2$ & 0.64 & $2.28 \mathrm{E}+12$ & 0.33 & 0.04 & $5.96 \mathrm{E}+11$ & 0.99 \\
\hline 114.313 & 0 & $5 / 2$ & 874,794 & $3 / 2$ & 0.99 & $5.02 \mathrm{E}+12$ & 0.93 & 1.01 & $5.48 \mathrm{E}+12$ & 1.05 \\
\hline 114.879 & 16,725 & $3 / 2$ & 887,203 & $5 / 2$ & 1.33 & $1.08 \mathrm{E}+13$ & 0.93 & 1.24 & $9.24 \mathrm{E}+12$ & 1.06 \\
\hline 114.880 & 0 & $5 / 2$ & 870,470 & $7 / 2$ & 1.46 & $1.47 \mathrm{E}+13$ & 0.93 & 1.37 & $1.24 \mathrm{E}+13$ & 1.06 \\
\hline 115.632 & 16,725 & $3 / 2$ & 881,539 & $3 / 2$ & 0.98 & $4.76 \mathrm{E}+12$ & 0.93 & 1.01 & $5.32 \mathrm{E}+12$ & 1.09 \\
\hline 115.661 & 0 & $5 / 2$ & 864,592 & $5 / 2$ & 1.30 & $9.98 \mathrm{E}+12$ & 0.92 & 1.22 & $8.76 \mathrm{E}+12$ & 1.10 \\
\hline 116.541 & 16,725 & $3 / 2$ & 874,794 & $3 / 2$ & 0.60 & $1.93 \mathrm{E}+12$ & 0.31 & 0.02 & $5.32 \mathrm{E}+11$ & 1.17 \\
\hline 117.943 & 16,725 & $3 / 2$ & 864,592 & $5 / 2$ & -0.86 & $6.64 \mathrm{E}+10$ & 0.04 & -1.37 & $2.14 \mathrm{E}+10$ & 1.33 \\
\hline 133.390 & 0 & $5 / 2$ & 749,681 & $3 / 2$ & -1.67 & $7.97 \mathrm{E}+09$ & 0.01 & -1.91 & $4.72 \mathrm{E}+09$ & 1.54 \\
\hline 134.190 & 0 & $5 / 2$ & 745,212 & $3 / 2$ & -1.37 & $1.57 \mathrm{E}+10$ & 0.04 & -1.31 & $1.79 \mathrm{E}+10$ & 0.99 \\
\hline 135.729 & 16,725 & $3 / 2$ & 753,489 & $1 / 2$ & -0.73 & $6.78 \mathrm{E}+10$ & 0.05 & -0.85 & $5.20 \mathrm{E}+10$ & 1.04 \\
\hline 136.434 & 16,725 & $3 / 2$ & 749,681 & $3 / 2$ & -2.05 & $3.16 \mathrm{E}+09$ & 0.00 & -2.24 & $2.10 \mathrm{E}+09$ & 1.16 \\
\hline 137.271 & 16,725 & $3 / 2$ & 745,212 & $3 / 2$ & -1.38 & $1.46 \mathrm{E}+10$ & 0.02 & -1.71 & $6.88 \mathrm{E}+09$ & 0.97 \\
\hline 138.529 & 0 & $5 / 2$ & 721,870 & $5 / 2$ & -1.85 & $4.89 \mathrm{E}+09$ & 0.01 & -1.98 & $3.71 \mathrm{E}+09$ & 0.97 \\
\hline 138.816 & 16,725 & $3 / 2$ & 737,104 & $1 / 2$ & -1.16 & $2.42 \mathrm{E}+10$ & 0.04 & -1.48 & $1.17 \mathrm{E}+10$ & 0.82 \\
\hline 140.126 & 0 & $5 / 2$ & 713,643 & $5 / 2$ & -2.15 & $2.43 E+09$ & 0.00 & -2.17 & $2.32 \mathrm{E}+09$ & 1.32 \\
\hline 140.569 & 0 & $5 / 2$ & 711,392 & $5 / 2$ & -2.63 & $7.98 \mathrm{E}+08$ & 0.00 & -3.73 & $6.30 \mathrm{E}+07$ & 0.11 \\
\hline 141.032 & 16,725 & $3 / 2$ & 725,785 & $1 / 2$ & -2.96 & $3.71 \mathrm{E}+08$ & 0.00 & -2.87 & $4.62 \mathrm{E}+08$ & 0.36 \\
\hline 141.094 & 0 & $5 / 2$ & 708,748 & $7 / 2$ & -1.40 & $1.33 \mathrm{E}+10$ & 0.01 & -1.62 & $8.00 \mathrm{E}+09$ & 1.14 \\
\hline 141.545 & 16,725 & $3 / 2$ & 723,216 & $1 / 2$ & -1.42 & $1.27 \mathrm{E}+10$ & 0.05 & -1.28 & $1.76 \mathrm{E}+10$ & 0.98 \\
\hline 142.046 & 0 & $5 / 2$ & 703,997 & $7 / 2$ & -0.91 & $4.11 \mathrm{E}+10$ & 0.10 & -0.86 & $4.52 \mathrm{E}+10$ & 1.00 \\
\hline 142.504 & 0 & $5 / 2$ & 701,735 & $5 / 2$ & -1.97 & $3.53 \mathrm{E}+09$ & 0.00 & -1.70 & $6.54 \mathrm{E}+09$ & 0.96 \\
\hline 142.622 & 0 & $5 / 2$ & 701,155 & $5 / 2$ & -1.76 & $5.67 \mathrm{E}+09$ & 0.00 & -2.61 & $8.10 \mathrm{E}+08$ & 0.82 \\
\hline 143.112 & 0 & $5 / 2$ & 698,751 & $5 / 2$ & -3.32 & $1.58 \mathrm{E}+08$ & 0.00 & -5.19 & $2.14 \mathrm{E}+06$ & 0.74 \\
\hline 143.382 & 0 & $5 / 2$ & 697,440 & $3 / 2$ & -1.89 & $4.17 \mathrm{E}+09$ & 0.01 & -2.22 & $1.98 \mathrm{E}+09$ & 0.90 \\
\hline 143.477 & 0 & $5 / 2$ & 696,975 & $3 / 2$ & -1.22 & $1.98 \mathrm{E}+10$ & 0.07 & -1.17 & $2.15 E+10$ & 1.01 \\
\hline 143.489 & 16,725 & $3 / 2$ & 713,643 & $5 / 2$ & -1.63 & $7.63 E+09$ & 0.01 & -1.76 & $5.71 \mathrm{E}+09$ & 1.10 \\
\hline 143.954 & 16,725 & $3 / 2$ & 711,392 & $5 / 2$ & -2.32 & $1.56 \mathrm{E}+09$ & 0.00 & -2.32 & $1.56 \mathrm{E}+09$ & 1.28 \\
\hline 144.081 & 0 & $5 / 2$ & 694,056 & $5 / 2$ & -1.87 & $4.38 \mathrm{E}+09$ & 0.02 & -1.89 & $4.09 \mathrm{E}+09$ & 1.02 \\
\hline 144.654 & 0 & $5 / 2$ & 691,306 & $3 / 2$ & -1.02 & $3.04 \mathrm{E}+10$ & 0.11 & -0.74 & $5.80 \mathrm{E}+10$ & 1.00 \\
\hline 144.769 & 0 & $5 / 2$ & 690,757 & $5 / 2$ & -1.36 & $1.38 \mathrm{E}+10$ & 0.05 & -1.10 & $2.47 \mathrm{E}+10$ & 0.98 \\
\hline 145.098 & 0 & $5 / 2$ & 689,190 & $3 / 2$ & -1.24 & $1.82 \mathrm{E}+10$ & 0.04 & -2.45 & $1.14 \mathrm{E}+09$ & 1.32 \\
\hline 145.150 & 16,725 & $3 / 2$ & 705,669 & $1 / 2$ & -0.19 & $2.07 \mathrm{E}+11$ & 0.63 & -0.19 & $2.00 \mathrm{E}+11$ & 1.01 \\
\hline 145.323 & 0 & $5 / 2$ & 688,121 & $3 / 2$ & -0.53 & $9.35 E+10$ & 0.21 & -0.49 & $1.02 \mathrm{E}+11$ & 1.01 \\
\hline 145.397 & 0 & $5 / 2$ & 687,770 & $7 / 2$ & -1.91 & $3.90 \mathrm{E}+09$ & 0.01 & -2.49 & $1.02 \mathrm{E}+09$ & 0.98 \\
\hline 145.715 & 0 & $5 / 2$ & 686,273 & $5 / 2$ & -2.05 & $2.78 \mathrm{E}+09$ & 0.01 & -2.36 & $1.38 \mathrm{E}+09$ & 1.23 \\
\hline 145.788 & 16,725 & $3 / 2$ & 702,652 & $1 / 2$ & -2.39 & $1.29 \mathrm{E}+09$ & 0.01 & -1.98 & $3.28 \mathrm{E}+09$ & 0.97 \\
\hline 145.983 & 16,725 & $3 / 2$ & 701,735 & $5 / 2$ & -0.22 & $1.89 \mathrm{E}+11$ & 0.18 & -0.07 & $2.65 \mathrm{E}+11$ & 1.00 \\
\hline 146.107 & 16,725 & $3 / 2$ & 701,155 & $5 / 2$ & -1.14 & $2.28 \mathrm{E}+10$ & 0.02 & -2.28 & $1.66 \mathrm{E}+09$ & 1.23 \\
\hline 146.148 & 0 & $5 / 2$ & 684,240 & $7 / 2$ & -1.44 & $1.13 E+10$ & 0.10 & -1.04 & $2.82 \mathrm{E}+10$ & 0.98 \\
\hline 146.413 & 0 & $5 / 2$ & 682,998 & $3 / 2$ & -0.89 & $4.01 \mathrm{E}+10$ & 0.29 & -0.90 & $3.89 \mathrm{E}+10$ & 0.98 \\
\hline 146.448 & 0 & $5 / 2$ & 682,838 & $5 / 2$ & -1.33 & $1.46 \mathrm{E}+10$ & 0.07 & -1.78 & $5.03 E+09$ & 1.03 \\
\hline 146.534 & 0 & $5 / 2$ & 682,437 & $3 / 2$ & -1.63 & $7.22 \mathrm{E}+09$ & 0.03 & -3.15 & $2.19 \mathrm{E}+08$ & 2.37 \\
\hline 146.622 & 16,725 & $3 / 2$ & 698,751 & $5 / 2$ & -1.40 & $1.24 \mathrm{E}+10$ & 0.01 & -2.40 & $1.24 \mathrm{E}+09$ & 1.22 \\
\hline 146.904 & 16,725 & $3 / 2$ & 697,440 & $3 / 2$ & -3.93 & $3.67 \mathrm{E}+07$ & 0.00 & -5.03 & $2.94 \mathrm{E}+06$ & 0.16 \\
\hline 147.005 & 16,725 & $3 / 2$ & 696,975 & $3 / 2$ & -1.80 & $4.89 \mathrm{E}+09$ & 0.01 & -1.65 & $6.76 \mathrm{E}+09$ & 0.96 \\
\hline 147.381 & 16,725 & $3 / 2$ & 695,239 & $1 / 2$ & -1.23 & $1.82 \mathrm{E}+10$ & 0.36 & -1.19 & $1.98 \mathrm{E}+10$ & 1.02 \\
\hline 147.416 & 0 & $5 / 2$ & 678,351 & $5 / 2$ & -1.69 & $6.28 \mathrm{E}+09$ & 0.02 & -3.74 & $5.56 \mathrm{E}+07$ & 0.55 \\
\hline 147.619 & 0 & $5 / 2$ & 677,421 & $3 / 2$ & -0.11 & $2.37 \mathrm{E}+11$ & 0.65 & -0.05 & $2.68 \mathrm{E}+11$ & 1.00 \\
\hline 147.638 & 16,725 & $3 / 2$ & 694,056 & $5 / 2$ & -0.67 & $6.56 \mathrm{E}+10$ & 0.35 & -0.73 & $5.58 \mathrm{E}+10$ & 1.00 \\
\hline 147.734 & 0 & $5 / 2$ & 676,893 & $7 / 2$ & -1.39 & $1.24 \mathrm{E}+10$ & 0.03 & -2.25 & $1.73 \mathrm{E}+09$ & 1.11 \\
\hline 147.956 & 0 & $5 / 2$ & 675,878 & $7 / 2$ & 0.05 & $3.45 \mathrm{E}+11$ & 0.47 & 0.14 & $4.19 \mathrm{E}+11$ & 1.00 \\
\hline 148.005 & 0 & $5 / 2$ & 675,652 & $5 / 2$ & -0.30 & $1.53 \mathrm{E}+11$ & 0.38 & -0.11 & $2.33 \mathrm{E}+11$ & 0.98 \\
\hline 148.240 & 16,725 & $3 / 2$ & 691,306 & $3 / 2$ & -1.17 & $2.08 \mathrm{E}+10$ & 0.11 & -0.58 & $7.92 \mathrm{E}+10$ & 0.98 \\
\hline 148.333 & 0 & $5 / 2$ & 674,159 & $3 / 2$ & -0.79 & $4.95 \mathrm{E}+10$ & 0.26 & -1.59 & $7.60 \mathrm{E}+09$ & 0.95 \\
\hline 148.361 & 16,725 & $3 / 2$ & 690,757 & $5 / 2$ & -0.36 & $1.31 \mathrm{E}+11$ & 0.25 & -0.41 & $1.16 \mathrm{E}+11$ & 1.01 \\
\hline 148.707 & 16,725 & $3 / 2$ & 689,190 & $3 / 2$ & -0.81 & $4.65 E+10$ & 0.23 & -5.18 & $1.99 \mathrm{E}+06$ & 1.08 \\
\hline 148.943 & 16,725 & $3 / 2$ & 688,121 & $3 / 2$ & -0.31 & $1.47 \mathrm{E}+11$ & 0.52 & -0.32 & $1.41 \mathrm{E}+11$ & 1.00 \\
\hline
\end{tabular}

radial functions and the expansion coefficients $c_{\mathrm{k}}$ are optimized to self-consistency [20,22].

In the present work, the spectroscopic configurations, i.e. $4 s^{2} 4 p^{6} 4 d^{9} \quad(J=3 / 2,5 / 2)$, for the even parity, and $4 s^{2} 4 p^{5} 4 d^{10}$, $4 s^{2} 4 p^{6} 4 d^{8} 5 p$ and $4 s^{2} 4 p^{6} 4 d^{8} 4 f(J=1 / 2-7 / 2)$, for the odd parity, were used as reference configurations to generate all the CSFs forming the basis set by single and double electron excitations to $4 \mathrm{~s}, 4 \mathrm{p}, 4 \mathrm{~d}, 4 \mathrm{f}, 5 \mathrm{~s}, 5 \mathrm{p}, 5 \mathrm{~d}$ and $5 \mathrm{f}$ orbitals. This led to a total number of 770596 CSFs, ensuring that the most important core- valence and valence-valence correlations were taken into account. The orbitals were then optimized using the extended average level (EAL) option of GRASP2K $[20,21]$ in separated Dirac-Hartree-Fock (DHF) calculations that included all the states of non-relativistic electronic configurations as follows:

- All the core orbitals, i.e. $1 \mathrm{~s}$ to $3 \mathrm{~d}$, along with the orbitals $4 \mathrm{~s}$, $4 \mathrm{p}$ and $4 \mathrm{~d}$ were obtained by an EAL DHF optimization on the ground configuration $4 \mathrm{~d}^{9}$; 
Table 3 (continued)

\begin{tabular}{|c|c|c|c|c|c|c|c|c|c|c|}
\hline \multirow[t]{2}{*}{$\lambda(\AA)^{\mathrm{a}}$} & \multirow{2}{*}{$\begin{array}{l}\text { Lower level }^{\mathrm{b}} \\
\mathrm{E}\left(\mathrm{cm}^{-1}\right)\end{array}$} & \multicolumn{3}{|c|}{ Upper level $^{\mathrm{b}}$} & \multicolumn{3}{|l|}{ HFR } & \multicolumn{3}{|c|}{ MCDHF } \\
\hline & & $\mathrm{J}$ & $\mathrm{E}\left(\mathrm{cm}^{-1}\right)$ & $\mathrm{J}$ & $\log g f$ & $\mathrm{gA}\left(\mathrm{s}^{-1}\right)$ & $\mathrm{CF}^{\mathrm{c}}$ & $\log g f$ & $\mathrm{gA}\left(\mathrm{s}^{-1}\right)$ & $\mathrm{B} / \mathrm{C}^{\mathrm{d}}$ \\
\hline 149.021 & 0 & $5 / 2$ & 671,045 & $5 / 2$ & -0.31 & $1.47 \mathrm{E}+11$ & 0.50 & -0.21 & $1.82 \mathrm{E}+11$ & 0.98 \\
\hline 149.358 & 0 & $5 / 2$ & 669,531 & $7 / 2$ & -0.19 & $1.93 E+11$ & 0.61 & -0.41 & $1.13 \mathrm{E}+11$ & 1.02 \\
\hline 149.583 & 0 & $5 / 2$ & 668,525 & $5 / 2$ & -0.64 & $6.87 \mathrm{E}+10$ & 0.45 & -0.82 & $4.40 \mathrm{E}+10$ & 0.99 \\
\hline 149.682 & 16,725 & $3 / 2$ & 684,807 & $1 / 2$ & -1.78 & $5.00 \mathrm{E}+09$ & 0.00 & -3.93 & $3.50 \mathrm{E}+07$ & 0.15 \\
\hline 150.089 & 16,725 & $3 / 2$ & 682,998 & $3 / 2$ & -0.04 & $2.71 \mathrm{E}+11$ & 0.49 & -0.03 & $2.71 \mathrm{E}+11$ & 0.99 \\
\hline 150.125 & 16,725 & $3 / 2$ & 682,838 & $5 / 2$ & -0.70 & $5.94 \mathrm{E}+10$ & 0.18 & -0.78 & $4.83 \mathrm{E}+10$ & 1.01 \\
\hline 150.215 & 16,725 & $3 / 2$ & 682,437 & $3 / 2$ & -1.96 & $3.24 \mathrm{E}+09$ & 0.01 & -2.55 & $8.44 \mathrm{E}+08$ & 0.85 \\
\hline 150.444 & 16,725 & $3 / 2$ & 681,425 & $1 / 2$ & -1.22 & $1.76 \mathrm{E}+10$ & 0.03 & -1.13 & $2.14 \mathrm{E}+10$ & 0.99 \\
\hline 150.544 & 0 & $5 / 2$ & 664,256 & $5 / 2$ & -0.08 & $2.44 \mathrm{E}+11$ & 0.38 & -0.15 & $2.03 E+11$ & 0.99 \\
\hline 151.021 & 0 & $5 / 2$ & 662,160 & $3 / 2$ & -1.36 & $1.29 \mathrm{E}+10$ & 0.10 & -1.62 & $6.88 \mathrm{E}+09$ & 0.99 \\
\hline 151.143 & 16,725 & $3 / 2$ & 678,351 & $5 / 2$ & -1.82 & $4.42 \mathrm{E}+09$ & 0.01 & -3.18 & $1.92 \mathrm{E}+08$ & 0.68 \\
\hline 151.291 & 16,725 & $3 / 2$ & 677,704 & $1 / 2$ & -1.55 & $8.27 \mathrm{E}+09$ & 0.14 & -1.72 & $5.54 \mathrm{E}+09$ & 1.08 \\
\hline 151.356 & 16,725 & $3 / 2$ & 677,421 & $3 / 2$ & -1.66 & $6.32 \mathrm{E}+09$ & 0.03 & -1.07 & $2.46 \mathrm{E}+10$ & 0.97 \\
\hline 151.747 & 0 & $5 / 2$ & 658,993 & $7 / 2$ & -0.51 & $8.94 \mathrm{E}+10$ & 0.24 & -0.52 & $8.56 \mathrm{E}+10$ & 0.99 \\
\hline 151.762 & 16,725 & $3 / 2$ & 675,652 & $5 / 2$ & -0.81 & $4.46 \mathrm{E}+10$ & 0.28 & -0.87 & $3.86 \mathrm{E}+10$ & 1.00 \\
\hline 152.058 & 0 & $5 / 2$ & 657,645 & $5 / 2$ & -0.41 & $1.12 \mathrm{E}+11$ & 0.35 & -0.46 & $9.84 \mathrm{E}+10$ & 0.99 \\
\hline 152.107 & 16,725 & $3 / 2$ & 674,159 & $3 / 2$ & -2.06 & $2.51 \mathrm{E}+09$ & 0.02 & -2.92 & $3.44 \mathrm{E}+08$ & 0.97 \\
\hline 152.830 & 16,725 & $3 / 2$ & 671,045 & $5 / 2$ & -1.32 & $1.37 \mathrm{E}+10$ & 0.06 & -1.15 & $2.00 \mathrm{E}+10$ & 1.02 \\
\hline 152.848 & 0 & $5 / 2$ & 654,245 & $3 / 2$ & -1.86 & $3.90 \mathrm{E}+09$ & 0.31 & -2.61 & $6.84 \mathrm{E}+08$ & 5.48 \\
\hline 153.421 & 16,725 & $3 / 2$ & 668,525 & $5 / 2$ & -2.46 & $9.86 \mathrm{E}+08$ & 0.01 & -4.54 & $8.04 \mathrm{E}+06$ & 0.27 \\
\hline 154.433 & 16,725 & $3 / 2$ & 664,256 & $5 / 2$ & -1.33 & $1.31 \mathrm{E}+10$ & 0.08 & -1.26 & $1.52 \mathrm{E}+10$ & 1.00 \\
\hline 154.588 & 0 & $5 / 2$ & 646,880 & $7 / 2$ & -1.96 & $3.07 \mathrm{E}+09$ & 0.13 & -2.18 & $1.82 \mathrm{E}+09$ & 0.98 \\
\hline 154.680 & 0 & $5 / 2$ & 646,494 & $5 / 2$ & -1.13 & $2.05 E+10$ & 0.19 & -1.22 & $1.65 \mathrm{E}+10$ & 1.00 \\
\hline 154.934 & 16,725 & $3 / 2$ & 662,160 & $3 / 2$ & -1.29 & $1.41 \mathrm{E}+10$ & 0.08 & -1.35 & $1.22 \mathrm{E}+10$ & 1.00 \\
\hline 155.248 & 0 & $5 / 2$ & 644,130 & $3 / 2$ & -2.25 & $1.56 \mathrm{E}+09$ & 0.01 & -1.15 & $1.98 \mathrm{E}+10$ & 1.44 \\
\hline 156.300 & 16,725 & $3 / 2$ & 656,520 & $1 / 2$ & -2.58 & $7.12 \mathrm{E}+08$ & 0.01 & -3.03 & $2.52 \mathrm{E}+08$ & 0.86 \\
\hline 156.858 & 16,725 & $3 / 2$ & 654,245 & $3 / 2$ & -2.18 & $1.79 \mathrm{E}+09$ & 0.07 & -2.26 & $1.46 \mathrm{E}+09$ & 1.34 \\
\hline 158.923 & 0 & $5 / 2$ & 629,234 & $3 / 2$ & -1.09 & $2.14 \mathrm{E}+10$ & 0.01 & -2.22 & $1.62 \mathrm{E}+09$ & 1.13 \\
\hline 158.972 & 0 & $5 / 2$ & 629,040 & $7 / 2$ & -2.22 & $1.60 \mathrm{E}+09$ & 0.02 & -2.34 & $1.18 \mathrm{E}+09$ & 0.97 \\
\hline 159.387 & 16,725 & $3 / 2$ & 644,130 & $3 / 2$ & -1.63 & $6.21 \mathrm{E}+09$ & 0.03 & -2.93 & $3.08 \mathrm{E}+08$ & 4.45 \\
\hline 163.263 & 16,725 & $3 / 2$ & 629,234 & $3 / 2$ & -2.01 & $2.43 \mathrm{E}+09$ & 0.01 & -1.64 & $5.92 \mathrm{E}+09$ & 1.00 \\
\hline
\end{tabular}

a Ritz wavelengths computed using the experimental energy level values.

b Experimental energy levels from [12].

c Cancellation factor (see text).

d Ratio between the transition rates calculated using the Babushkin (B) and the Coulomb (C) gauges (see text).

- The orbitals $4 \mathrm{f}, 5 \mathrm{~s}, 5 \mathrm{p}, 5 \mathrm{~d}$ and $5 \mathrm{f}$ were optimized on the configurations $4 d^{8} 4 f, 4 d^{8} 5 s, 4 d^{8} 5 p, 4 d^{8} 5 d$ and $4 d^{8} 5 f$, respectively.

So as to improve the calculations, the orbitals $4 \mathrm{p}, 4 \mathrm{~d}, 4 \mathrm{f}$ and $5 p$ were then re-optimized, keeping frozen all the other orbitals, in an extended optimized level (EOL) variational procedure using the basis of 770596 CSFs and by minimizing an energy functional built from the first 99 ASFs of each $\mathrm{J}^{\Pi}$ symmetry.

Finally, higher-order relativistic effects, such as the Breit interaction, QED self-energy and vacuum polarization effects [20], were incorporated in the relativistic configuration interaction (RCI) step of the GRASP2K program [21].

The MCDHF energy levels are compared with the available experimental values, as well as with the HFR ones, in Table 2.

\section{Radiative decay parameters}

In Table 3, we give the oscillator strengths and transition probabilities computed in the present work using both methods described hereabove for a set of 92 Xe X transitions with HFR log gf-values greater than -4.0 . These lines appear in the extreme ultraviolet spectral region from 110 to $164 \AA$. When comparing the results obtained with our two computational approaches, one can notice an overall good agreement (within 20-30\%). This general agreement is illustrated in Fig. 1 where oscillator strengths are compared for all transitions listed in Table 3. When looking into more details, we found that, for the whole set of 92 transitions, the average ratio $\mathrm{gA}_{\mathrm{MCDHF}} / \mathrm{gA}_{\mathrm{HFR}}$ was equal to $0.965 \pm 1.438$, where the uncertainty represents the deviation from the mean. The rather large value of the latter is essentially due to transitions affected by strong cancellation effects in the HFR calculations or significant

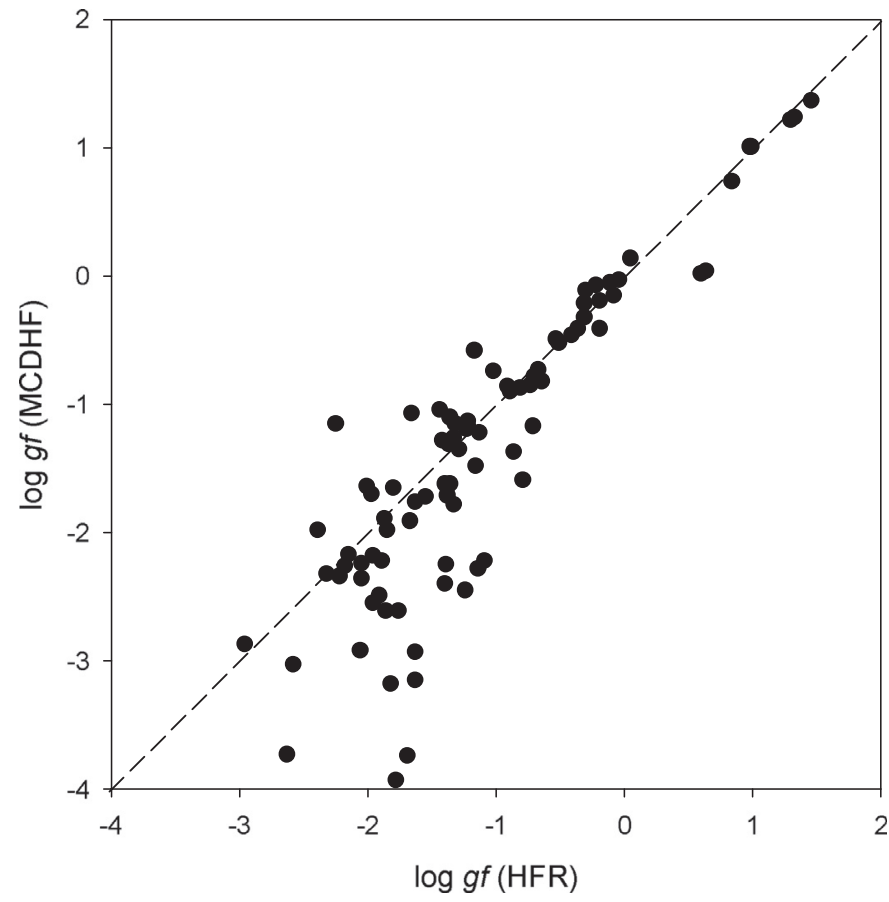

Fig. 1. Comparison between the log gf-values obtained in the present work with the multiconfiguration Dirac-Hartree-Fock method (MCDHF) and the pseudorelativistic Hartree-Fock approach $(\mathrm{HFR}+\mathrm{CPOL})$ for Xe X spectral lines. Only transitions with $\log \mathrm{gf}>-4$ are shown in the figure. 
discrepancies between the Babushkin and Coulomb gauges in the MCDHF calculations. Indeed, when considering only transitions for which the HFR line strengths are such that the cancellation factors (CF), as defined by Cowan [19], are larger than 0.05 and, at the same time, the MCDHF results for which the Babushkin gauge do not differ by more than $20 \%$ from the Coulomb one, the scattering between HFR and MCDHF transition probabilities is considerably reduced, the average ratio $\mathrm{gA}_{\mathrm{MCDHF}} / \mathrm{g} \mathrm{A}_{\mathrm{HFR}}$ being then found to be equal to $1.004 \pm 0.617$. In order to make the use of our new radiative data easier, the cancellation factors obtained in the HFR calculations, as well as the ratio between the Babushkin and Coulomb MCDHF results, are also reported for all Xe X transitions in Table 3.

\section{Conclusion}

Using two different theoretical approaches, it has been possible to obtain, for the first time, a set of reliable radiative parameters, i.e. oscillator strengths and transition probabilities, for $92 \mathrm{Xe}$ $\mathrm{X}$ spectral lines appearing in the extreme ultraviolet region. These new results, for which an overall agreement within $20-30 \%$ was found between both methods, complete those we recently published for Xe V-Xe IX ions and are expected to be useful for applications in other scientific areas, such as laboratory plasma physics, where xenon ions are supposed to present a particular interest.

\section{Acknowledgements}

PP and PQ are respectively Research Director and Research Associate of the Belgian Fund for Scientific Research F.R.S.-FNRS. Financial support from this organization is gratefully acknowledged. EBM is grateful to Belgian colleagues for their hospitality during his stay at Mons University.

\section{References}

[1] Milora SL, Houlberg WA, Lengyel LL, Mertens V. Pellet fuelling. Nucl. Fus. 1995;35:657.

[2] Reznichenko PV, Vinyar IV, Kuteev BV. An injector of xenon macroscopic pellets for quenching the fusion reaction in a tokamak. Techn. Phys. 2000;45:174.
[3] Beiersdorfer P. Highly charged ions in magnetic fusion plasmas: research opportunities and diagnostic necessities. J. Phys. B 2015;48:144017.

[4] Almandos JR, Raineri M. Spectral analysis of moderately charged rare-gas atoms. Atoms. 2017;5:12.

[5] Duchowicz R, Schinca D, Gallardo M. New analysis for the assignment of UV-visible ionic Xe laser lines. IEEE J. Quant. Elect. 1994;30:155-9.

[6] Gallardo M, Raineri M, Reyna Almandos JG, Sobral H, Callegari F. Revised and extended analysis in four times ionized xenon, Xe V. J. Quant. Spectrosc. Radiat. Transf. 1999;61:319-27.

[7] Sobral H, Schinca D, Gallardo M, Duchowicz R. Time dependent study of a multi-Ionic xenon plasma. J. Appl. Phys. 1999;85:69-73.

[8] Sobral H, Raineri M, Schinca D, Gallardo M, Duchowicz R. Excitation mechanisms and characterization of a multi-Ionic xenon laser. IEEE J. Quantum Electron. 1999:35:1308-13.

[9] Raineri M, Lagorio C, Padilla S, Gallardo M, Reyna Almandos J. Weighted oscillator strengths for the Xe IV spectrum. At. Data Nucl. Data Tables 2008:94:140-59.

[10] Reyna Almandos J, Bredice F, Raineri M, Gallardo M. Spectral analysis of ionized noble gases and implications for astronomy and laser studies. Phys. Scr. T 2009;134:014018.

[11] Gallardo M, Raineri M, Reyna Almandos J, Biémont E. New energy levels, calculated lifetimes and transition probabilities in Xe IX. J. Phys. B: At. Mol. Opt. Phys. 2011;44:045001.

[12] Saloman EB. Energy levels and observed spectral lines of xenon, Xe I through Xe LIV. J. Phys. Chem. Ref. Data 2004;33:765.

[13] Biémont E, Quinet P, Zeippen CJ. Transition probabilities in Xe V. Phys. Scr. 2005; $71: 163$.

[14] Biémont E, Buchard V, Garnir HP, Lefèbvre PH, Quinet P. Radiative lifetime and oscillator strength determinations in Xe VI. Eur. Phys. J. D 2005;33:181.

[15] Biémont E, Clar M, Fivet V, Garnir HP, Palmeri P, Quinet P, Rostohar D. Lifetime and transition probability determination in xenon ions. The cases of Xe VII and Xe VIII. Eur. Phys. J. D 2007;44:23.

[16] Garnir HP, Enzonga Yoca S, Quinet P, Biémont E. Lifetime and transition probability determination in Xe IX. J. Quant. Spectr. Rad. Transf. 2009;110:284.

[17] Quinet P, Palmeri P, Biémont E, McCurdy MM, Rieger G, Pinnington EH, Wickliffe ME, Lawler JE. Experimental and theoretical lifetimes, branching fractions and oscillator strengths in Lu II. Mon. Not. R. Astr. Soc. 1999;307:934.

[18] Quinet P, Palmeri P, Biémont E, Li Z S, Zhang Z G, Svanberg S. Radiative lifetime measurements and transition probability calculations in lanthanide ions. J. Alloys Comp 2002;344:255.

[19] Cowan RD. The theory of atomic structure and spectra. Berkeley: University of California Press; 1981.

[20] Grant IP. Relativistic quantum theory of atoms and molecules. Theory and computation. New York: Springer; 2007.

[21] Jönsson P, Gaigalas G, Bieron J, Froese Fischer C, Grant I P. New version: GRASP2K relativistic atomic structure package. Comput. Phys. Commun. 2013;184:2197.

[22] Froese Fischer C, Godefroid M, Brage T, Jönsson P, Gaigalas G. Advanced multiconfiguration methods for complex atoms: I. energies and wave function. J. Phys. B; At. Mol. Opt. Phys. 2016;49:182004. 